\title{
Variáveis Individuais e Familiares na Psoríase: Um Estudo com Doentes e Parceiros ${ }^{1}$
}

\author{
Laura Brito ${ }^{2}$ \\ Maria da Graça Pereira \\ Universidade do Minho
}

\begin{abstract}
RESUMO - Este trabalho avalia morbilidade, qualidade de vida, ajustamento de casal, coping familiar e imagem corporal em 101 doentes com psoríase e 78 parceiros. Os resultados revelaram uma relação positiva entre as variáveis dos doentes e parceiros no que diz respeito ao ajustamento, coping e morbilidade. Pior qualidade de vida e imagem corporal no doente estão associadas a mais morbilidade no parceiro. Os doentes em tratamento combinado e fototerapia apresentaram pior qualidade de vida e insatisfação com a imagem corporal. Melhor ajustamento de casal e menor ansiedade no parceiro predizem ajustamento de casal no doente. Satisfação com a imagem corporal prevê melhor qualidade de vida no doente. Os resultados enfatizam a necessidade de envolver os parceiros nos programas de intervenção. As implicações são apresentadas.
\end{abstract}

Palavras-chave: psoríase, morbilidade, qualidade de vida, ajustamento conjugal, coping, imagem corporal.

\section{Individual and Family Variables in Psoriasis: A Study with Patients and Partners}

\begin{abstract}
In this paper the relationship between morbility, quality of life, marital adjustment, family coping and body image in 101 psoriatic patients and 78 partners was investigated. The results showed a positive relationship between patients and partners variables on dyadic adjustment, coping and morbidity. Negative quality of life and body image in patients are associated to more morbidity in partners. Patients in combined treatment and phototherapy showed lower quality of life and less satisfaction with body image. Better dyadic adjustment and less anxiety in partners predict higher adjustment in patients. Satisfaction with body image predicts better quality of life in patients. The results of this study emphasize the need to involve partners in program's interventions. Clinical implications of the results are presented.
\end{abstract}

Key words: Psoriasis, morbility, quality of life, dyadic adjustment, coping, body image.

O comportamento dos sujeitos influencia o aparecimento de mudanças no estado de saúde e pode alterar a evolução da doença. A influência dos factores psicossociais é fundamental quando se trata das estratégias de coping para lidar com a doença, na adesão terapêutica e, de uma forma geral, na redução e na prevenção de problemas psicológicos associados à condição médica (Papadopoulos \& Walker, 2003). É neste sentido que a Psicologia da Saúde pode fornecer informação pertinente para melhor compreender a adaptação a uma doença crónica quer a nível individual quer familiar.

A psoríase é uma doença de pele, geneticamente determinada, que consiste essencialmente na renovação exagerada da epiderme por multiplicação das células espinhosas, associada a inflamação dérmica, o que conduz a lesões eritematopapulodescamativas (Koo, Lee, Lee, \& Lebwohl, 2004). As lesões são, em regra, de contornos bem limitados e localizam-se preferencialmente nas superfícies extensoras e no couro cabeludo. Contudo, muitas variantes são possíveis, atingindo exclusivamente as unhas, as palmas e plantas ou as pregas e mucosas, até às formas generalizadas a toda a superfície cutânea. As lesões são em regra assintomáticas, mas quando são secas e fissuradas podem ser dolorosas e

1 A editoria associada da revista optou por manter a grafia do português de Portugal.

2 Endreço para Correspondência: Universidade do Minho - Escola de Psicologia Braga, Portugal. E-mail:britolaura@hotmail.com. surgem em surtos de intensidade variável intercalados por períodos de remissão (Griffiths \& Barker, 2007).

A psoríase afeta 1 a $3 \%$ da população mundial. Atinge igualmente homens e mulheres, embora o início seja mais precoce nas mulheres. Existem dois picos de idade de prevalência: entre os 15 e os 30 e após os 50 anos (Resende \& Azevedo, 2002). Em Portugal, numa amostra total de 3320 doentes com psoríase de gravidade clínica moderada a grave verificou-se que em $64 \%$ dos casos o primeiro surto ocorre antes dos 30 anos e com prevalência superior na população masculina. A baixa laboral média, devido à psoríase, foi de 3 meses/ano com grande impacto na qualidade de vida dos doentes, reflectindo-se no facto de os mesmos estarem dispostos a ceder, em média, 2 anos da sua vida para se verem livres da doença (Cabral et al., 2005). Além de factores de índole genética, outros cofactores intrínsecos e/ou extrínsecos (psicológicos, stress, frio, medicamentos, tabagismo e bebidas alcoólicas) estão envolvidos no seu aparecimento e evolução (Fry, 2004).

$\mathrm{Na}$ psoríase, $40-90 \%$ dos pacientes apresentam morbilidade psicológica (Zachariae et al., 2002). Também, Choi e Koo (2003) verificaram que uma grande percentagem de doentes refere ter níveis moderados a extremos de ansiedade e depressão, quer em períodos de exacerbação, quer em remissão. Diversos estudos têm descrito uma associação da psoríase com ansiedade, distúrbios de personalidade e 
comportamento, depressão e ideação suicida (Bahmer, Kuhl, \& Bahmer, 2007; Esposito, Saraceno, Giunta, Maccarone, \& Chimenti, 2006; Russo, Ilchef, \& Cooper, 2004; Schmitt \& Ford, 2007).

A dificuldade emergente das reacções dos outros face às lesões de pele desfigurativas é percepcionada pelos doentes como factor de grande impacto negativo (Schmid-Ott, Stephan, \& Werfel, 2003). Por conseguinte, esta experiência de rejeição poderá conduzir a uma percepção negativa da imagem corporal e a comportamentos de evitamento e receio aquando uma possível exposição da imagem corporal (Walker \& Papadopoulos, 2005; Magin et al., 2009).

Quanto à qualidade de vida, diversas investigações descrevem, em termos individuais, que a psoríase causa impacto negativo nas diversas dimensões, incluindo as actividades diárias, lazer (tais como desporto e praia), trabalho/escola e relações pessoais (contactos sociais e relações íntimas) (Finlay, 2001; Leibovici et al., 2010). Este impacto no bem-estar geral está consequentemente associado a uma perda de produtividade no trabalho, níveis mais elevados de stresse psicológico e insatisfação com o tratamento (Schmitt \& Ford, 2006; 2007).

Tal como outras doenças crónicas, a psoríase implica alterações na vida do casal nomeadamente, mais encargos domésticos, disponibilidade para acompanhar o parceiro no tratamento, limitações nos planos de férias e actividades de lazer, limitações nas actividades diárias e alterações ao nível da intimidade (Basra \& Finlay, 2007; Eghlileb, Davies, \& Finlay, 2007; Gordon \& Perrone, 2004; Sampogna, Gisondi, Tabolli, \& Abeni, 2007). Além disso, e no caso especifico da psoríase, existe ainda desconforto físico, desfiguração, embaraço e estigma social (Kent, 2000). De facto, sentimentos de vergonha podem mudar o sentido de self do indivíduo e alterar o modo como o doente aborda as relações de casal provocando insegurança emocional nas relações existentes (Tantleff-Dunn \& Gokee, 2002). Assim, a alteração dos hábitos de rotina do casal pela doença e pelo tratamento efectuado pode ameaçar a natureza relacional. De um modo geral, 26\% dos doentes afirmaram que a psoríase interferiu na relação emocional com o parceiro (Walker \& Papadopoulos, 2005). Deste modo, as mudanças que esta afecção cutânea acarretará e as adaptações que a família irá realizar dependem dos recursos que dispõe, de como a doença começou e o significado que atribui ao acontecimento, isto é do coping familiar (Papadopoulos \& Walker, 2003).

Para que o tratamento seja eficaz é importante fornecer orientações gerais quanto à doença, individualizando o tratamento conforme as características de cada paciente. Deste modo, existem quatro tipos de tratamento: tópico, fototerapia, sistémico e combinados (fototerapia associada a sistémico, tópico associado a sistémico) (Gottlieb, 2005). Em termos de tratamento, Richards et al. (2003) verificaram que a fototerapia provocava melhoria na mobilidade, menos stress e menos sintomas da doença. Contudo, não ocorreram diferenças significativas nos índices de ansiedade e depressão, representações da doença e coping. Por outro lado, os tratamentos tópico (Skevington, Bradshaw, Hepplewhite, Dawkes \& Lovell, 2006) e sistémico (Gardinal, Ammoury \& Paul, 2009) estavam associados a maior satisfação e melhor qualidade de vida.
Em suma, a revisão da literatura tem revelado a importância das variáveis psicossociais, como a morbilidade psicológica (depressão e ansiedade), a qualidade de vida, o ajustamento do casal, o coping familiar e a imagem corporal, no processo de adaptação psicossocial à psoríase. São, contudo, poucos os estudos que têm focado, além dos doentes, os parceiros. Assim, o presente estudo, de uma forma geral, pretende conhecer a relação entre as variáveis psicológicas na psoríase, em doentes e parceiros.

De acordo com a literatura, espera-se que as variáveis psicológicas nos doentes estejam correlacionadas com as mesmas variáveis nos parceiros. Ao nível do tipo de tratamento, espera-se que os doentes que receberam tratamento tópico, sistémico ou fototerapia apresentem melhor qualidade de vida. Finalmente, espera-se que as variáveis psicológicas, incluindo as variáveis dos parceiros, exerçam um contributo diferente no ajustamento de casal e qualidade de vida nos doentes com psoríase.

\section{Método}

\section{Participantes}

A amostra da presente investigação foi constituída pelo universo de usuários do Hospital de São Marcos em Braga, especificamente da consulta externa de Dermatologia e de uma clínica privada de Braga. Trata-se de um estudo transversal com uma amostra de conveniência de 101 pacientes com psoríase clinicamente definida e 78 parceiros respectivos.

Quanto às características sociodemográficas, relativamente ao sexo dos participantes, verifica-se que no grupo dos doentes a amostra é constituída por 51 homens e 50 mulheres. A amostra dos parceiros é constituída por 37 homens e 41 mulheres (alguns doentes não tem parceiro(a)). No grupo de doentes, a média das idades é de 44 anos (mínima 18 e máxima 80). Quanto aos parceiros, a média das idades é de 46 anos (mínima 18 e máxima 80). Quanto às habilitações académicas, $35.6 \%$ dos doentes têm o ensino primário ou preparatório e $17.8 \%$ completaram o ensino superior. Nos parceiros, $41 \%$ dos sujeitos têm o ensino primário ou preparatório e $10.3 \%$ completaram o ensino superior. Quanto ao tipo de tratamento actual, a terapia de fototerapia foi a mais utilizada (39.6\%), seguida de tratamentos combinados (27.7\%), tópicos $(24.8 \%)$ e sistémicos $(7.9 \%)$.

\section{Procedimentos}

Após aprovação da investigação no comitê de ética do hospital, realizou-se uma reunião no serviço de dermatologia de modo a obter o apoio dos médicos no estudo. Neste sentido, os doentes foram contactados telefonicamente no dia anterior à consulta e convidados a participar na investigação. No dia da avaliação, o paciente e o parceiro responderam aos questionários individualmente em salas separadas após lhes ser apresentado os objectivos específicos do estudo e completaram o consentimento informado. $\mathrm{O}$ investigador, durante a avaliação visitou cada uma das salas no sentido de esclarecer 
dúvidas. O preenchimento teve a duração aproximada de 2030 minutos. A participação no estudo foi voluntária.

\section{Instrumentos}

Os pacientes completaram os seguintes instrumentos: Hospital Anxiety and Depression Scale; Body Image Scale; Psoriasis Disability Index; Revised Dyadic Adjustment Scale e Family Crisis Oriented Personal Evaluation Scale. Os instrumentos para os parceiros foram os seguintes: State Trait Anxiety Inventory; Inventário de Beck para a Depressão; Revised Dyadic Adjustment Scale e Family Crisis Oriented Personal Evaluation Scale.

Todos os instrumentos foram adaptados e validados na presente amostra, à excepção do instrumento que avalia o coping familiar dado não existir um número suficiente de sujeitos. A Family Crisis Oriented Personal Evaluation Scale (F-COPES) (McCubbin, Larsen \& Olson, 1991), versão adaptada (Mendes \& Relvas, 1999), é constituída por 29 itens que identificam a resolução de um problema e estratégias comportamentais utilizadas pela família. Possui 5 subescalas: obtenção de suporte social, reenquadramento, procura de suporte espiritual, mobilização da família para obtenção e aceitação de ajuda e avaliação passiva. Quanto à cotação, os itens baseiam-se segundo a escala de Likert de 5 -pontos, sendo que 1 «discordo totalmente» e 5 «concordo totalmente». As dimensões de estratégias de coping familiar no F-COPES são avaliadas mediante o recurso ao valor total e aos resultados nas sub-escalas. Resultados elevados estão associados a um elevado recurso de estratégias de coping familiar eficazes. A fidelidade do instrumento, neste estudo, indica que o alfa obtido por dimensão foi: obtenção de suporte social $(0,80)$; reenquadramento $(0,70)$; procura de suporte espiritual $(0,81)$; mobilização da família para obtenção e aceitação de ajuda $(0,75)$, avaliação passiva $(0,52)$ e o F-COPES - total é de $(0,86)$. Na amostra de parceiros, o coeficiente de fidelidade para o F-COPES - total é de $(0,88)$ e por dimensão foi de: obtenção de suporte social $(0,83)$; reenquadramento $(0,66)$; procura de suporte espiritual $(0,84)$; mobilização da família para obtenção e aceitação de ajuda $(0,77)$ e avaliação passiva $(0,69)$.

A Body Image Scale (BIS): (Hopwood, Fletcher, Lee \& Ghazal, 2001), versão adaptada (Cotrim \& Pereira, 2008), é constituída por 10 itens que integram itens afectivos, comportamentais e cognitivos. Os resultados em cada um dos itens podem ir de 0 (de modo algum) a 3 (muito), reflectindo a gravidade do impacto que a doença teve na sua autoimagem. $\mathrm{O}$ alfa obtido foi $(0,93)$. A Hospital Anxiety and Depression Scale (HADS) (Zigmond \& Snaith, 1983), versão adaptada (Brito \& Pereira, 2008), avalia os níveis de ansiedade e depressão em doentes com patologia física e em tratamento ambulatório. É constituída por 14 itens divididos em duas sub-escalas: ansiedade ( 7 itens) e depressão ( 7 itens). O modo de resposta varia de 0 (baixo) a 3 (elevado), numa escala Likert de 4 pontos. Os autores consideram que a gravidade da ansiedade e da depressão podem ser classificadas como "normal" (0-7), leve (8-10), moderada (11-15) e severa (1621). O coeficiente de fidelidade para o HADS - total é de $(0,91)$, na escala depressão é de 0,91 e na ansiedade 0,87 .
Outro instrumento selecionado foi o Psoriasis Disability Index (PDI) (Finlay \& Coles, 1995), versão de investigação (Pereira \& Brito, 2008), utilizado para avaliar o impacto da psoríase. Quanto mais elevada a pontuação total, pior qualidade de vida. Para cada item, o máximo que se pode obter é de 3 , sendo a cotação de 0 a 3 (0 - nada; 1 - ligeiramente; 2- bastante; 3 - muito). O coeficiente de fidelidade para o PDI - total é de $(0,90)$ e para as subescalas actividades diárias $(0,83)$ e relacionamento com os outros $(0,90)$.

A Revised Dyadic Adjustment Scale (R-DAS) (Busby, Christensen, Crabe \& Larson, 1995), versão adaptada (Pereira, 2003), destina-se a avaliar a qualidade do relacionamento conjugal. Embora pretenda avaliar a qualidade do relacionamento conjugal no geral, esta escala divide-se em três subescalas que podem ser analisadas separadamente: consenso diádico, satisfação diádica e coesão diádica. No consenso diádico a cotação varia de 5 (sempre de acordo) a 0 (sempre em desacordo). Na satisfação diádica, varia de 0 (sempre) a 5 (nunca) e na coesão, o item 11 varia de 0 (nunca) a 4 (todos os dias) e os restantes itens 12, 13 e 14 variam de 0 (nunca) a 5 (frequentemente). Valores elevados indicam melhor relacionamento conjugal. O coeficiente de fidelidade para o R-DAS - total foi de $(0,90)$ e para as subescalas consenso $(0,92)$, satisfação $(0,87)$ e coesão $(0,67)$. $\mathrm{Na}$ amostra dos parceiros, o coeficiente de fidelidade para o R-DAS - total é de $(0,90)$, e para as subescalas: consenso $(0,92)$, satisfação $(0,84)$ e coesão $(0,73)$.

O Beck Depression Inventory (BDI-II) (Beck, Steer \& Brown, 1996), versão adaptada (McIntyre \& Araújo-Soares, 1999), aborda sintomas da depressão. As pontuações podem ser categorizadas da seguinte maneira: menos de $10=$ não deprimido; 10-19 = levemente deprimido, 20-25 = moderadamente deprimido; 26 ou mais $=$ gravemente deprimido. $\mathrm{O}$ alfa obtido foi de $(0,89)$.

O State Trait Anxiety Inventory (STAI) (Spielberger, Gorush, Lushene, Vagg \& Jacobs, 1983), versão adaptada (McIntyre \& McIntyre, 1995), é composto por duas subescalas: a escala de ansiedade estado (STAI form Y-1) e a escala de ansiedade traço (STAI form Y-2). A cotação de cada item varia de 1 a 4 pontos, correspondendo 1 ao grau mínimo e 4 ao grau máximo de ansiedade. Os primeiros 20 itens correspondem à avaliação da escala de estado de ansiedade e os últimos 20 itens da escala de traço de ansiedade. Quanto maior o resultado (traço/estado) maior é a ansiedade traço ou ansiedade estado conforme o caso. Os valores de alfa encontrados foram de $(0,96)$ para a escala de estado e de $(0,94)$ para a escala traço.

\section{Análise de Dados}

Para testar a relação entre as variáveis, utilizou-se a Correlação de Pearson. Para avaliar a influência da variável clínica tipo de tratamento recorreu-se ao teste Kruskal-Wallis, dado não se verificarem os pressupostos da estatística paramétrica. Para determinar os melhores preditores do ajustamento de casal e da qualidade de vida recorreu-se a análises de regressão segundo o modelo de regressão linear múltipla com o método enter. 


\section{Resultados}

Mediante os resultados da Tabela 1, verificamos que maior morbilidade psicológica e insatisfação com a imagem corporal, no doente, estão associadas a mais morbilidade psicológica, pior ajustamento de casal e menos recursos de coping familiar nos parceiros (à excepção da depressão do doente que não está associada ao coping do parceiro). Melhor ajustamento de casal e mais recursos de coping familiar no doente estão associados a menor morbilidade psicológica, melhor ajustamento de casal e mais recursos de coping familiar no parceiro. Verificamos ainda que pacientes com pior qualidade de vida global têm parceiros com níveis elevados de sintomatologia depressiva e ansiosa. Contudo, não se verificou uma relação entre qualidade de vida do doente e ansiedade estado, ajustamento de casal ou coping familiar no parceiro.

Verificamos que existem diferenças estatisticamente significativas ao nível da imagem corporal, qualidade de vida em geral e na dimensão especifica de actividades diárias, bem como ao nível do ajustamento de casal global e na dimensão coesão, e finalmente no coping familiar e subescalas obtenção de suporte social e mobilização da família para obtenção e aceitação de ajuda. Os grupos de tratamentos combinado e de fototerapia $(p=0,041)$ apresentam maior insatisfação com a imagem corporal em comparação com os outros grupos. $\mathrm{O}$ grupo de tratamento combinado apresenta pior qualidade de vida em geral $(p=0,031)$ e pior na subescala actividades diárias $(\mathrm{p}=0,012)$, comparando com os outros. Os grupos de tratamento tópico, fototerapia e sistémico apresentam melhor ajustamento de casal $(p=0,032)$, coesão $(p=0,020)$, mais coping familiar $(\mathrm{p}=0,005)$ e mais recursos em termos de obtenção de suporte social $(p=0,004)$ e mobilização da família para obtenção e aceitação de ajuda $(\mathrm{p}=0,005)$ do que o tratamento combinado (ver Tabela 2).

Não se verificaram diferenças significativas nas variáveis morbilidade psicológica, ajustamento de casal e coping familiar nos parceiros, de doentes com psoríase, em função do tratamento dos doentes.
Verificamos que a variável imagem corporal é a variável do estudo que exerce um contributo significativo para a qualidade de vida, explicando 31\% da variância. Deste modo, quanto mais satisfação com a imagem corporal, melhor a qualidade de vida total (Tabela 3).

Quanto ao ajustamento de casal, verificamos que a imagem corporal no primeiro modelo é significativa, isto é, quanta mais insatisfação com a imagem corporal pior o ajustamento de casal nos doentes. Contudo, esta variável deixa de ser significativa quando são acrescentadas as variáveis psicológicas dos parceiros. As variáveis ajustamento de casal (parceiro) e ansiedade estado (parceiro) são as variáveis do estudo que exercem um contributo significativo para o ajustamento de casal no doente explicando $69 \%$ da variância. Assim, quanto mais positivo o ajustamento de casal e menor sintomatologia de ansiedade estado do parceiro, melhor o ajustamento de casal do doente (ver Tabela 3).

\section{Discussão}

Encontrou-se uma relação positiva entre níveis de ajustamento de casal, morbilidade psicológica e coping familiar entre pacientes e parceiros. Verificamos uma relação negativa entre ajustamento de casal, nos parceiros, e as variáveis morbilidade psicológica e imagem corporal no doente, por um lado, e entre coping familiar e as variáveis ansiedade e imagem corporal, por outro. Os resultados estão de acordo com a literatura (Northouse, Mood, Templin, Mellon, \& George, 2000), confirmando que uma condição crónica não afecta apenas o indivíduo, mas também a família. Entre doentes e parceiros, a ansiedade encontra-se inversamente relacionada com o coping familiar e o ajustamento de casal e estes, por sua vez, positivamente relacionados entre si (coping familiar e ajustamento de casal). Tais resultados podem dever-se à vivência em comum do impacto que estas sintomatologias têm na dinâmica conjugal. No caso da psoríase, sentimentos de ansiedade e depressão vão diminuir

Tabela 1. Coeficientes de Correlação de Pearson entre Morbilidade Psicológica, Imagem Corporal, Qualidade de Vida, Ajustamento do Casal e Coping Familiar em Doentes e Parceiros

\begin{tabular}{|c|c|c|c|c|c|c|}
\hline Variáveis & 1 & 2 & 3 & 4 & 5 & 6 \\
\hline \multirow[t]{2}{*}{ Depressão } & $.346^{* *}$ & $.411 * * *$ & $.423 * * *$ & $.295^{* *}$ & $.516^{* * *}$ & $-.299 * *$ \\
\hline & & . & & . & & \\
\hline \multirow[t]{2}{*}{ Ansiedade Estado } & $.232 *$ & $.360 * *$ & $.422 * * *$ & .218 & $-.601 * * *$ & $-.256^{*}$ \\
\hline & & . & & & . & . \\
\hline Ansiedade Traço & $.280 *$ & $.437 * * *$ & $.429 * * *$ & $.282 *$ & $-.559 * * *$ & $-.316^{* *}$ \\
\hline \multirow[t]{2}{*}{ Ajustamento de Casal } & $-.249 *$ & $-.330 * *$ & $.409 * * *$ & -.190 & $.786^{* * *}$ & $.393^{* * *}$ \\
\hline & & & & & $\cdot$ & . \\
\hline Coping Familiar & -.209 & $-.280 *$ & $-.296^{* *}$ & -.218 & $.417 * * *$ & $.505^{* * *}$ \\
\hline
\end{tabular}

Nota: Nas correlações as variáveis dos doentes ( $\mathrm{n}=101)$ são apresentadas na horizontal e as dos parceiros na vertical (n=78). $1=$ depressão; $2=$ ansiedade; $3=$ imagem corporal; $4=$ qualidade de vida; $5=$ ajustamento de casal; $6=$ coping familiar $* \mathrm{p} \leq .05 * * \mathrm{p} \leq .01 * * * \mathrm{p} \leq .001$. 
Tabela 2. Resultados Significativos (Teste Kruskall-Wallis) ao Nível da Morbilidade Psicológica, Imagem Corporal, Ajustamento do Casal, Qualidade de Vida e Coping Familiar em Função do Tipo de Tratamento

\begin{tabular}{|c|c|c|c|c|c|c|}
\hline \multirow[t]{2}{*}{ Variáveis Dependentes } & \multicolumn{4}{|c|}{$\begin{array}{c}\text { Tratamento } \\
\text { (Posição Média) }\end{array}$} & \multirow[t]{2}{*}{$\chi^{2}$} & \multirow[t]{2}{*}{$\mathbf{p}$} \\
\hline & $\begin{array}{l}\text { Tópico } \\
(n=25)\end{array}$ & $\begin{array}{l}\text { Fotod. } \\
(n=40)\end{array}$ & $\begin{array}{l}\text { Sistémico } \\
\quad(n=8)\end{array}$ & $\begin{array}{r}\text { Comb. } \\
(n=28)\end{array}$ & & \\
\hline Imagem Corporal & 38.68 & 53.60 & 43.19 & 60.52 & 8.283 & $.041 *$ \\
\hline Actividades Diárias (QV) & 40.58 & 49.04 & 42.63 & 65.50 & 10.925 & $.012 *$ \\
\hline Qualidade de Vida Total & 41.98 & 49.61 & 40.94 & 63.91 & 8.862 & $.031 * *$ \\
\hline Coesão (Ajust. Casal) & 60.60 & 53.15 & 58.81 & 37.13 & 9.841 & $.020^{*}$ \\
\hline Ajustamento Total & 57.34 & 54.69 & 60.69 & 37.30 & 8.817 & $.032 *$ \\
\hline $\begin{array}{l}\text { Obtenção de Suporte Social } \\
\text { (Cop. Familiar) }\end{array}$ & 52.62 & 57.59 & 68.56 & 35.13 & 13.274 & $.004 * *$ \\
\hline $\begin{array}{l}\text { Mobilização da Família para a } \\
\text { Obtenção e Aceitação de Ajuda } \\
\text { (Cop. Familiar) }\end{array}$ & 50.48 & 58.45 & 67.94 & 35.98 & 12.717 & $.005^{* *}$ \\
\hline Coping Familiar Total & 53.86 & 56.86 & 68.56 & 35.05 & 13.021 & $.005^{* *}$ \\
\hline
\end{tabular}

Nota. $\mathrm{QV}=$ qualidade de vida; Ajust. Casal = ajustamento de casal; Cop. Familiar = coping familiar; Fotod. $=$ fotodinâmico; Comb. $=$ combinado $* \mathrm{p} \leq .05 * * \mathrm{p} \leq .01 * * * \mathrm{p} \leq .001$.

Tabela 3. Preditores da Qualidade de Vida e Ajustamento de Casal nos Doentes $(\mathrm{N}=101)$

\begin{tabular}{|c|c|c|c|c|c|c|}
\hline \multirow{3}{*}{ Variáveis } & \multicolumn{6}{|c|}{ Preditores } \\
\hline & \multicolumn{3}{|c|}{ Qualidade de Vida } & \multicolumn{3}{|c|}{ Ajustamento de Casal } \\
\hline & $\beta$ & $\mathrm{p}$ & $\Delta \mathrm{R} 2$ & $\beta$ & $\mathrm{p}$ & $\Delta \mathrm{R} 2$ \\
\hline Step 1 & & & .304 & & & .304 \\
\hline Depressão & .322 & .078 & & -.217 & .415 & \\
\hline Ansiedade & .254 & .202 & & -.332 & .256 & \\
\hline Imagem Corporal & .338 & $.019 *$ & & -.655 & $.002 * *$ & \\
\hline Step 2 & & & .007 & & & .392 \\
\hline Depressão & .307 & .103 & & -.097 & .597 & \\
\hline Ansiedade & .220 & .289 & & -.215 & .294 & \\
\hline Imagem Corporal & .347 & $.023 *$ & & -.246 & .103 & \\
\hline Depressão (parceiro) & .039 & .832 & & -.142 & .428 & \\
\hline Ans.-Estado (parceiro) & -.094 & .422 & & -.232 & $.048 *$ & \\
\hline Ans.-Traço) (parceiro) & .097 & .541 & & .250 & .117 & \\
\hline Ajust. Casal (parceiro) & & & & .592 & $.000 * * *$ & \\
\hline Adjusted R2 & & .253 & & & .666 & \\
\hline
\end{tabular}

Nota: Ans. = ansiedade; Ajust. Casal = ajustamento de casal.

$* \mathrm{p} \leq .05 * * \mathrm{p} \leq .01 * * * \mathrm{p} \leq .001$. 
o número de contactos e actividades sociais do próprio e da díade. Estes sentimentos e as suas repercussões vão ser sentidos pelo parceiro, podendo levar ao desenvolvimento dos mesmos sintomas. Pelo contrário, relações íntimas e carinhosas podem influenciar positivamente a saúde do paciente pelo fornecimento de suporte emocional. Assim, quando os casais ampliam a sua experiência de intimidade, melhorando a qualidade da relação e a coesão do casal, conseguem recorrer a estratégias de coping que permitem lidar melhor com a doença (Jerant, von Friederichs-Fitzwater, \& Moore, 2005; Rolland, 1994; Walker \& Papadopoulos, 2005). Quanto à relação estabelecida entre os níveis de morbilidade psicológica do doente e o ajustamento de casal do parceiro verifica-se que elevados níveis de morbilidade estão associados a pior percepção de ajustamento de casal por parte do parceiro. Estes dados parecem apoiar dados de outros estudos que referem que um aumento da morbilidade psicológica diminui a satisfação conjugal. Tal como D’Ardenne (2004) referiu muitos pacientes com diagnóstico de doença crónica têm de realizar ajustamentos substanciais às suas próprias vidas, o que envolve os seus parceiros. Assim, podemos inferir que a psoríase faz exigências na vida do casal (Rolland, 1994) que implicam reorganizações dos papéis. Deste modo, o presente estudo, sustenta as afirmações de Frangos e Kimball (2008) e Eghlileb e colaboradores (2007) quando referem que as dificuldades em manter contactos sociais podem conduzir a morbilidade psicológica no parceiro pelo facto deste ter de lidar com os efeitos negativos da psoríase que prejudicam o seu bem-estar.

No presente estudo, verificou-se que elevados níveis de morbilidade estão associados a menos recursos de coping familiar e maior morbilidade no doente está associada a maior morbilidade no parceiro. Estes resultados vão em conformidade com o estudo de Richards, Chong, Mason e Griffiths (2002) que encontraram relações positivas significativas entre doentes e parceiros quanto à morbilidade psicológica. A relação estabelecida entre morbilidade psicológica no doente e parceiro poderá advir da dificuldade de controlo bem como de sentimentos de impotência sentidos por parte do parceiro. A possível falta de informação sobre a doença (dimensão tipológica) e a ausência de recursos poderão ser condicionantes negativos e reforçadores destes sentimentos. Deste modo, o sentimento de falta de controlo sobre a condição médica, por informações e representações negativas da doença, por parte do doente, poderão ser agravadas pelo próprio parceiro, uma vez que este também não possui conhecimentos suficientes e/ ou correctos. A falta de controlo da doença poderá aumentar os sentimentos de mal-estar psicológico, físico bem como o receio de intimidade. No entanto, uma excepção foi verificada no coping familiar do parceiro que não se relacionou com a depressão do doente. Este resultado é curioso pois verifica-se para a depressão mas não para a ansiedade. Estudos futuros deverão incluir doentes em diferentes fases da doença pois talvez a depressão do doente possa ser mais sensível numa fase em que o doente é mais velho, afectando mais a díada conjugal nessa fase, e neste estudo, a maior parte dos doentes foi diagnosticado entre os 16 e 30 anos.

Verificou-se que pior qualidade de vida no doente estava associada a mais sintomatologia depressiva e ansiedade traço no parceiro. Tais resultados evidenciam que a morbilidade do parceiro afecta a qualidade de vida do doente e vice-versa. De facto, o suporte do parceiro é essencial para o ajustamento à doença (Pistrang, Barker, \& Rutter, 1997) e quando o parceiro se encontra deprimido ou ansioso mais dificuldades terá a apoiar o doente podendo assim afectar a qualidade de vida deste. Como afirma Hayes e Koo (2010), quando as lesões estão exacerbadas, mesmo que seja só visível para o paciente, sentimentos de imperfeição podem alterar actividades normais do casal bem como a intimidade. Deste modo, tais atitudes poderão provocar no próprio parceiro sentimentos de impotência e sintomatologia depressiva e ansiosa (Tantleff-Dunn \& Gokee, 2002). Pelo contrário, os parceiros que demonstrarem aceitação e transmitirem emoções de valorização e atracção propiciam uma melhor qualidade de vida (Kellet, 2002). Assim, os resultados revelam que a psoríase não interfere somente com a vida diária e o funcionamento social dos pacientes, mas parece também interferir na morbilidade psicológica dos parceiros. De facto, Eghlileb e colaboradores (2007) concluíram que as categorias assinaladas pelos parceiros como mais deterioradas devido à doença (incluindo o impacto psicológico) estavam mais fortemente relacionadas com a qualidade de vida do paciente do que com a gravidade clinica da psoríase.

No entanto, a qualidade de vida não se encontrou associada à ansiedade (estado), ajustamento de casal e coping familiar do parceiro. Tal poderá ter a ver com o facto de a psoríase ser uma doença crónica que visa mais o controlo dos sintomas e adaptação à doença permitindo que as famílias se reorganizem ao longo do tempo. Considerando que a média de duração da doença neste estudo é de 19 anos, os doentes encontravam-se numa fase mais estável no sistema familiar ao nível da estrutura i.e., regras, rotinas, e papeis relacionados com a doença crónica.

Em relação ao tratamento, o grupo de tratamentos combinado e de fototerapia apresentam maior insatisfação com a imagem corporal em comparação com os outros grupos. $\mathrm{O}$ grupo de tratamento combinado apresenta pior qualidade de vida em geral e na subescala Actividades Diárias, comparando com os outros. Os grupos de tratamento tópico, fototerapia e sistémico apresentam melhor ajustamento de casal, melhor percepção de coesão do casal, mais coping familiar e mais recursos em termos de obtenção de suporte social e mobilização da família para obtenção e aceitação de ajuda. Estes resultados estão em conformidade com os estudos referidos (Gardinal et al., 2009; Richards et al., 2003; Skevington et al., 2006) que verificaram que doentes submetidos a tratamento tópico, sistémico ou fototerapia apresentavam melhor qualidade de vida. Além disso, os autores verificaram que factores psicológicos relacionados com a imagem corporal, auto-estima e sentimentos negativos eram particularmente sensíveis a mudanças clínicas. No entanto, também se deverá dar atenção aos factores familiares, uma vez que verificamos que nas variáveis familiares, como por exemplo o coping familiar e o ajustamento de casal também ocorreram diferenças. Assim, estes resultados podem ser explicados pelo facto de se realizar um único tipo de tratamento não implicar tantos encargos domésticos, aumento nas despesas económicas, alteração da rotina diária ou de deslocação ao hospital para a realização do tratamento como refere Basra e Finlay (2007), em que uma das dificuldades evidenciadas pelos familiares 
era a sobrecarga no cuidar (54\%), aumento nas despesas da família $(30 \%)$ e o aumento significativo na rotina diária de limpeza da casa (42\%). Também no estudo de Eghlileb e colaboradores (2007), 70\% dos participantes descreveram que o tratamento dos seus parceiros ou familiares tem como consequência o aumento de trabalhos domésticos, a perda de tempo na aplicação do tratamento e $55 \%$ dos familiares evidenciaram disrupção social em consequência da falta de confiança social e de disponibilidade de tempo devido à prestação de cuidados e de tratamento.

Em termos de qualidade de vida, a imagem corporal é a única variável preditiva do modelo. Quanto mais negativa a percepção da imagem corporal maior o impacto negativo na qualidade de vida. Tais resultados podem indicar que a satisfação com a imagem corporal ou não, é um indicador do bem-estar. Por conseguinte, um dos aspectos que interfere no impacto social é a visibilidade das lesões, a qual está correlacionada negativamente com a qualidade de vida (Leibovici et al., 2010). O facto de a pele ser exposta aos outros e ser o cartão de visita remete a que estes doentes tentem, geralmente, esconder as lesões. Estas estratégias decorrem do receio de avaliações externas e comentários pouco agradáveis. Deste modo, a depressão e a ansiedade são algumas das repercussões que surgem no decurso da influência da doença (Walker \& Papadopoulos, 2005), bem como, uma diminuição na realização de tarefas diárias ou de lazer. Por conseguinte, mais dificuldades em torno das actividades diárias são resultantes de problemas emocionais tais como ansiedade e depressão (Wahl, Loge, Wiklund, \& Hanestad, 2000).

Em relação ao ajustamento de casal, no doente, os resultados obtidos no primeiro modelo são corroborados por estudos anteriores como de Walker e Papadopoulos (2005) que evidenciam que a satisfação com a imagem corporal influencia o relacionamento do casal. Anível global, as variáveis preditivas são o ajustamento de casal e ansiedade estado dos parceiros. Este resultado parece indicar que na díade conjugal a percepção de ajustamento, do parceiro, contribui para a percepção de ajustamento do doente. De facto, este equilíbrio poderá permitir que ambos os membros do casal consigam utilizar estratégias e recursos familiares para lidar com as dificuldades da doença crónica. A ansiedade contribui negativamente e tal resultado compreende-se intuitivamente dado que as exigências aos parceiros aumentam. Além disso, as responsabilidades, papéis, recursos e o próprio relacionamento requerem um ajustamento que poderá ser vivenciado com ansiedade face às mudanças exigidas (Rolland, 1994; Sampogna, Gisondi, Tabolli, \& Abeni, 2007).

Este estudo veio evidenciar a importância de inclusão das variáveis familiares no ajustamento psicossocial à doença e nas intervenções com doentes com psoríase. Deste modo, intervir no casal e, nomeadamente nos parceiros, permitirá um melhor ajustamento à doença e, consequentemente, a promoção duma melhor qualidade de vida com implicações directas na adesão terapêutica do doente (Basto, 2008). Contudo, os resultados devem ser lidos com algumas reservas dado o número de sujeitos da presente amostra ser reduzido e se inscrever na zona norte de Portugal e a um hospital central. Futuras investigações devem incluir as representações da doença e o suporte social, uma vez que estas variáveis poderão constituir-se como factores mediadores ou moderadores na psoríase. Seria também importante replicar este estudo com doentes em diferentes fases da doença.

A presente investigação reforça a importância do desenvolvimento de programas de intervenção psicológica que incluam o doente e o parceiro. Considerando que o parceiro é o principal sistema de apoio do paciente, torna-se crucial incluí-lo nos programas de intervenção psicológica mas também nas consultas médicas, sempre que possível, no sentido de melhorar a qualidade de vida do casal e à adaptação psoríase no doente.

\section{Referências}

Bahmer, J. A., Kuhl, J., \& Bahmer, F. A. (2007). How do personality systems interact in patients with psoriasis, atopic dermatitis and urticaria? Acta Dermato-Venereologica, 87, 317-324.

Basra, M. K. A., \& Finlay, A. Y. (2007). The family impact of skin diseases: The greater patient concept. British Journal of Dermatology, 156(5), 929-937.

Basto, L. (2008). Variáveis psicossociais na psoríase: um estudo com doentes e seus parceiros. Dissertação de Mestrado, Universidade do Minho, Braga, Portugal.

Beck, A. T, Steer, R. A. \& Brown, G. K. (1996). Manual for the Beck Depression Inventory-II. San Antonio, TX: Psychological Corporation.

Brito, L., \& Pereira, M. G. (2008). Morbilidade psicológica e imagem corporal em doentes com psoríase: estudo das características psicométricas do Hospital Anxiety and Depression Scale. In C. Machado, L. Almeida, M. Guisande, M. Gonçalves, \& V. Ramalho (Eds.), Livro de Actas da XIII Conferência Internacional Avaliação Psicológica: Formas e Contextos (pp. A129). Braga: Universidade do Minho.

Busby, D. M., Christensen, C., Crabe, D. R., \& Larson, J. H. (1995). A revision of the dyadic adjustment scale for use with distressed and non distressed couples: Construct hierarchy and multidimensional scales. Journal of Marital and Family Therapy, 21(3), 289-308.

Cabral, M. F., Baptista, A., Cabrita, J., Caetano, M., Cardoso, R., Cirne de Castro, J. L., Tellechea, O., Dessai, S., \& Vieira, R. (2005). Avaliação epidemiológica da psoríase moderada a grave em Portugal e Espanha. Psoríase Newsletter, 2.

Choi, J., \& Koo, J. Y. (2003). Quality of issues in psoriasis. Journal of the American Academy of Dermatology, 49 (suppl.), S57- 61.

Cotrim, H., \& Pereira, M. G. (2008). Imagem corporal, morbilidade psicológica e qualidade de vida em doentes com cancro colorectal: Estudos das características psicométricas do Body Image Scale. In C. Machado, L. Almeida, M. Guisande, M. Gonçalves \& V. Ramalho (Eds.), Livro de Actas da XIII Conferência Internacional Avaliação Psicológica: Formas e Contextos (pp. A130). Braga: Universidade do Minho.

D’Ardenne, P. (2004). The couple sharing long-term illness. Sexual and Relationship Therapy, 19(3), 291-308.

Eghlileb, A. M., Davies, E. E. G., \& Finlay, A. Y. (2007). Psoriasis has a major secondary impact on the lives of family members and partners. British Journal of Dermatology, 156(6), 12451250 . 
Esposito, M., Saraceno, R., Giunta, A., Maccarone, M., \& Chimenti, S. (2006). An Italian study on psoriasis and depression. Dermatology, 212, 123-127.

Finlay, A. Y. (2001). Psoriasis from the patient's point of view. Archives of Dermatology, 137, 352-353.

Finlay, A. Y., \& Coles, E. C. (1995). The effect of severe psoriasis on the quality of life of 369 patients. British Journal of Dermatology, 132(2), 236-44.

Frangos, J. E., \& Kimball, A. B. (2008). Divorce/marriage ratio in patients with psoriasis compared to patients with other chronic medical conditions. Journal of Investigative Dermatology, 128(suppl. 1), S87.

Fry, L. (2004). An atlas of psoriasis (2 ${ }^{\mathrm{a}}$ ed.). London: Taylor \& Francis.

Gardinal, I., Ammoury, A., \& Paul, C. (2009). Moderate to severe psoriasis: From topical to biological treatment. Journal of the European Academy of Dermatology and Venereology, 23, 1324-1326.

Gordon, P. A., \& Perrone, K. M. (2004). When spouses become caregivers: Counseling implications for younger couples. Journal of Rehabilitation, 70(2), 27-32.

Gottlieb, A. B. (2005). Therapeutic options in the treatment of psoriasis and atopic dermatitis. Journal of the American Academy of Dermatology, 53, s3-16.

Griffiths, C. E., \& Barker, J. N. (2007). Pathogenesis and clinical features of psoriasis. Lancet, 370, 263-271.

Hayes, J., \& Koo, J. (2010). Psoriasis: Depression, anxiety, smoking, and drinking habits. Dermatology Therapy, 23(2), 174-80.

Hopwood, P., Fletcher, I., Lee, A., \& Al Ghazal, S. (2001). A body image scale for use with cancer patients. European Journal of Cancer, 37, 189-197.

Jerant, A. F., Von Friederichs-Fitzwater, M. M., \& Moore, M. (2005). Patients' perceived barriers to active self-management of chronic conditions. Patient Education and Counseling, 57, 300-307.

Kellet, S. (2002). Shame-focused acne: A biopsychosocial conceptualisation and treatment rationale. In P. Gilbert \& J. Miles (Eds.), Body shame: Conceptualisation, research and treatment (pp. 135-154). East Sussex: Brunner-Routledge.

Kent, G. (2000). Understanding the experiences of people with disfigurements: An integration of four models of social and psychological functioning. Psychology, Health and Medicine, 5(2), 117-129.

Koo, J., Lee, E., Lee, C. S., \& Lebwohl, M. (2004). Psoriasis. Journal of the American Academy of Dermatology, 50, 613-622.

Leibovici, V., Canetti, L., Yahalomi, S., Cooper-Kazaz, R., Bonne, O., Ingber, A., \& Bachar, E. (2010). Well being, psychopathology and coping strategies in psoriasis compared with atopic dermatitis: A controlled study. Journal of the European Academy of Dermatology and Venereology, 24, 897-903.

Magin, P., Adams, J., Heading, G., Pond, D., \& Wayne, S. (2009). The psychological sequelae of psoriasis: Results of a qualitative study. Psychology, Health \& Medicine, 14(2), 150-161.

McCubbin, H. I., Larsen, A. S., \& Olson, D. H. (1991). F-COPES: Family Crisis Oriented Personal Evaluation Scales. In H. McCubbin \& H. Thompson (Eds.), Family assessment inventories for research and practice (2a ed., pp. 203-216). Madison, WI: University of Wisconsin-Madison.
McIntyre, L., \& McIntyre, S. (1995). State Trait Anxiety Inventory (STAI) - versão de investigação. Braga: Universidade do Minho.

McIntyre, M., \& Araújo-Soares, V. (1999). Inventário da Depressão de Beck: Estudo de validade numa amostra de doentes com dor crónica. In A.P. Soares, S. Araújo, \& S. Caires (Eds.), Livro de Actas da VII Conferência Internacional Avaliação Psicológica: Formas e Contextos (pp. 245-255). Braga: Universidade do Minho.

Mendes, F., \& Relvas, A. P. (1999). Family relationships and primary prevention of drug use on early adolescence. Valencia: IREFREA \& European Commission.

Northouse, L. L., Mood, D., Templin, T., Mellon, S., George, T. (2000). Couples' patterns of adjustment to colon cancer. Social Science \& Medicine, 50(2), 271-284.

Papadopoulos, L., \& Walker, C. (2003). Understanding skin problems. London: John Wiley \& Sons.

Pereira, M. G. (2003). Escala Revista de Ajustamento Diádico (RDAS) - Versão de Investigação. Braga: Universidade do Minho.

Pereira, M. G., \& Brito, L. (2008). Psoriasis Disability Index Versão de Investigação. Braga: Universidade do Minho.

Pistrang, N., Barker, C., \& Rutter, C. (1997). Social support as conversation: Analyzing breast cancer patient's interactions with their partners. Social Science and Medicine, 45(5), 773782.

Resende, C., \& Azevedo, F. (2002). Psoríase. Lisboa: Permanyer Portugal.

Richards, H. L., Chong, S. L. P., Mason, D. L., \& Griffiths, C. E. M. (2002). The impact of psoriasis on healthy partners patients with psoriasis. British Journal of Dermatology, 147(62), 40.

Richards, H. L., Fortune, D. G., Macelhone, K., Kirby, B., Main, C. J., \& Griffiths, C. E. M. (2003). The psychological impact of psoriasis: does successful treatment result in meaningful improvements in patients' well-being? Dermatology and Psychosomatics, 4, 116.

Rolland, J. S. (1994). Families, illness and disability: An integrative treatment model. New York: Basic Books.

Russo, P. A., Ilchef, R., \& Cooper, A. J. (2004). Psychiatric morbidity in psoriasis: A review. Australasian Journal of Dermatology, 45, 155-159.

Sampogna, F., Gisondi, P., Tabolli, S., \& Abeni, D. (2007). Impairment of sexual life in patients with psoriasis. Dermatology, 214, 144-150.

Schmid-Ott, G., Stephan, M., \& Werfel, T. (2003). Psoriasis. Dermatology and Psychosomatics, 4, 169-171.

Schmitt, J., \& Ford, D. E. (2010). Psoriasis is independently associated with psychiatric morbidity and adverse cardiovascular risk factors, but not with cardiovascular events in a population-based sample. Journal of the European Academy of Dermatology and Venereology, 24(8), 1-8.

Schmitt, J. M., \& Ford, D. E. (2006). Work limitations and productivity loss are associated with health-related quality of life but not with clinical severity in patients with psoriasis. Dermatology, 213 (2), 102-110.

Schmitt, J. M., \& Ford, D. E. (2007). Role of depression in quality of life for patients with psoriasis. Dermatology, 215, 17-27. 
Skevington, S. M., Bradshaw, J., Hepplewhite, A., Dawkes, K., \& Lovell, C. R. (2006). How does psoriasis affect quality of life? Assessing an Ingram-regimen outpatient programme and validating the WHOQOL-100. British Journal of Dermatology, 154, 680-691.

Spielberger, C. D., Gorush, R., Lushene, R., Vagg P.R., \& Jacobs, G. A. (1983). Manual for the State-Trait Anxiety Inventory. Palo Alto, CA: Consulting Psychologists Press.

Tantleff-Dunn, S., \& Gokee, J. L. (2002). Interpersonal influence on body image development. In T. F. Cash, T. Pruzinsky (Eds.), Body image: A Handbook of theory, research and clinical practice (pp.108-1124). London: Guildford press.

Wahl, A., Loge, J. H., Wiklund, I., \& Hanestad, B. R. (2000). The burden of psoriasis: A study concerning health-related quality of life among Norwegian adult patients with psoriasis compared with general population norms. Journal of the American Academy of Dermatology, 43(5), 803-808.
Walker, C., \& Papadopoulos, L. (2005). Psychodermatology: The psychological impact of skin disorders. New York: Cambridge University Press.

Zachariae, R., Zachariae, H., Blomqvist, K., Davidsson, S., Molin, L., Mork, C., \& Sigurgeirsson, B. (2002). Quality of life in 6497 Nordic patients with psoriasis. British Journal of Dermatology, 146(6), 1006-1016.

Zigmond, A. S., \& Snaith, R. P. (1983). The Hospital Anxiety and Depression Scale. Acta Psychiatrica Scandinavica, 67(6), 361-370.

Recebido em 30.04.2010

Primeira decisão editorial em 04.02.2011

Versão final em 01.08.2011

Aceito em 06.10.2011 